\title{
The Role of Apelin on the Alleviative Effect of Angiotensin Receptor Blocker in Unilateral Ureteral Obstruction-Induced Renal Fibrosis
}

\author{
Masashi Nishida Yasuko Okumura Tatsujiro Oka Kentaro Toiyama \\ Seiichiro Ozawa Toshiyuki Itoi Kenji Hamaoka \\ Department of Pediatric Cardiology and Nephrology, Kyoto Prefectural University of Medicine \\ Graduate School of Medical Science, Kyoto, Japan
}

\section{Key Words}

Apelin $\cdot$ APJ receptor $\cdot$ Angiotensin receptor blocker $\cdot$ Nitric oxide $\cdot$ Renal fibrosis $\cdot$ Unilateral ureteral obstruction

\begin{abstract}
Background: Apelin is a selective endogenous ligand of the APJ receptor, which genetically has closest identity to the angiotensin II type 1 receptor (AT-1). The effects of the apelin/APJ system on renal fibrosis still remain unclear. Methods: We examined the effects of the apelin/APJ system on renal fibrosis during AT-1 blockade in a mouse unilateral ureteral obstruction (UUO) model. Results: We obtained the following results: (1) At UUO day 7, mRNA expressions of apelin/APJ and phosphorylations of Akt/endothelial nitric oxide synthase (eNOS) in the UUO kidney were increased compared to those in the nonobstructed kidney. (2) AT-1 blockade by the treatment with losartan resulted in a further increase of apelin mRNA as well as phosphorylations of Akt/eNOS proteins, and this was accompanied by alleviated renal interstitial fibrosis, decreased myofibroblast accumulation, and a decreased number of interstitial macrophages. (3) Blockade of the APJ receptor by the treatment with F13A during losartan administration completely abrogated the effects of losartan in the activation of the Akt/eNOS pathway and the amelioration of renal fibrosis. (4) Inhibition of NOS by the treatment with L-NAME also resulted in a further increase in renal fibrosis compared to the control group. Conclusion: These results suggest that increased nitric oxide production through the apelin/APJ/Akt/eNOS pathway may, at least in part, contribute to the alleviative effect of losartan in UUO-induced renal fibrosis.
\end{abstract}

Copyright $\odot 2012$ S. Karger AG, Basel 


\section{Introduction}

Apelin is a selective endogenous ligand of the G protein-coupled receptor APJ and was originally isolated from bovine stomach extracts $[1,2]$. The APJ receptor was originally identified as a gene with closest identity to the angiotensin II type 1 receptor (AT-1) and was kept 'orphan' until the identification of apelin [1]. Both apelin and the APJ receptor were found to be distributed in a wide variety of tissues including the central nervous system, heart, lungs, and kidneys [3-7], and the apelin/APJ system has been shown to have relevant roles in the regulation of cardiovascular, gastrointestinal, and immune functions, bone physiology, fluid homeostasis, and embryonic development of the cardiovascular system [8-10]. Interestingly, in spite of a high homology between the APJ and the AT-1 receptors, and similar patterns of tissue expression for both receptors, previous reports showed opposing actions between the apelin/APJ system and the angiotensin II (Ang II)/AT-1 system in a number of physiologic and pathophysiologic settings [11-17]. Apelin mediates opposing actions to Ang II on vascular tone, blood pressure, and fluid homeostasis [11-17]. Furthermore, recent reports showed antifibrotic actions of apelin in the cardiovascular system that counteracts to Ang II $[17,18]$. However, the role of apelin in renal fibrosis, where the Ang II/AT-1 system has been proved to have critical roles $[19,20]$, has not yet been elucidated. Additionally, previous studies have shown that apelin acts to increase nitric oxide (NO) production in endothelial cells [21], and NO exerts tissue-protective effects in several pathological settings including renal fibrosis induced by unilateral ureteral obstruction (UUO) that is a nonimmunological model of progressive renal fibrosis $[22,23]$. Thus, in this study, we used a mouse UUO model and studied the role of apelin in renal fibrosis during the administration of angiotensin receptor blocker, and also the role of $\mathrm{NO}$ production on these changes.

\section{Materials and Methods}

\section{Experimental Protocol}

Experiments were performed on male C57BL/6 mice (10-12 weeks, 20-24 g). These mice were subjected to a complete UUO as previously described (day 0) [24]. Briefly, under sodium pentobarbital anesthesia, the middle portion of the left ureter was ligated and cut between the two ligated points. The mice were sacrificed at day 7 after surgery, and the obstructed kidneys were harvested and subjected to the studies. The mice were divided to a total of four groups as follows: (1) control, (2) losartan (a specific AT-1 receptor antagonist), (3) losartan + F13A (a specific APJ receptor antagonist), and (4) losartan + L-NAME [a NO synthase (NOS) inhibitor]. Mice of the losartan and L-NAME treatment groups were fed water containing $0.1 \mathrm{mg} / \mathrm{ml}$ (30 mg/kg per day) losartan (Merck \& Co. Inc., Rahway, N.J., USA) and/ or $0.1 \mathrm{mg} / \mathrm{ml} \mathrm{L-NAME} \mathrm{(Tocris} \mathrm{Cookson} \mathrm{Inc.,} \mathrm{Ellisville,} \mathrm{Mo.,} \mathrm{USA)} \mathrm{starting} 3$ days before UUO until day 7 after surgery. Mice in the F13A treatment group were injected intraperitoneally once a day with a daily dose of $150 \mu \mathrm{g} / \mathrm{kg}$ F13A (Phoenix Pharmaceuticals Inc., Burlingame, Calif., USA) diluted in $0.4 \mathrm{ml}$ saline or saline only as controls. The systolic blood pressure of the mice was measured by the tail-cuff method using MK-2000ST (Muromachi Kikai, Inc., Tokyo, Japan) at day 0 , day 3 , and day 7 after UUO. The experimental protocols were performed according to the regulations of the Kyoto Prefectural University of Medicine Animal Care Committee.

\section{RT-PCR Analysis}

Total RNA was isolated from the UUO kidneys using Sepasol (R)-RNA I Super (Nacalai Tesque, Inc., Kyoto, Japan), and RT-PCR for apelin, APJ receptor, and GAPDH mRNA was 
performed as previously described [16]. In brief, the sample RNA was reverse-transcribed using a High Capacity cDNA Archive kit (Applied Biosystems, Foster City, Calif., USA). PCR amplification was performed using Power SYBR ${ }^{\circledR}$ Green PCR Master Mix (Applied Biosystems). The forward and reverse primers for mouse apelin mRNA were 5'-CGAGTTGCAGCATGAATCTGAG-3' and 5'-TGTTCCATCTGGAGGCAACATC-3', respectively, and those for mouse APJ receptor were 5'-GTGGCCAATGCTCGGCTAA-3' and 5'-TGGAACGGAACACCATGACAG-3', whereas those for GAPDH were 5'-TGTGTCCGTCGTGGATCTGA-3' and 5'-TTGCTGTTGAAGTCGCAGGAG-3'.

\section{Western Blot Analysis}

Western blot analysis was performed as previously described [17]. In brief, the obstructed kidneys were lysed in lysis buffer containing $20 \mathrm{~mm}$ Tris- $\mathrm{HCl} \mathrm{pH}$ 8.0, $150 \mathrm{mM} \mathrm{NaCl}, 1 \mathrm{mM}$ EDTA, $100 \mathrm{~mm} \mathrm{NaF}, 1 \mathrm{mM} \mathrm{Na}_{3} \mathrm{VO}_{4}$, and 1\% proteinase inhibitor (Sigma-Aldrich Japan K.K., Tokyo, Japan). Lysates were centrifuged at $10,000 \mathrm{~g}$ for $10 \mathrm{~min}$, and supernatants (30 $\mu \mathrm{g}$ protein/lane) were separated by SDS-PAGE on a 10\% acrylamide gel. Gels were electroblotted onto a nitrocellulose membrane (GE Healthcare UK Ltd., Little Chalfont, UK). The membranes were blocked with $2 \%$ nonfat dry milk and incubated with either polyclonal rabbit anti-phospho-endothelial NOS (p-eNOS; Cell Signaling Technology Inc., Danvers, Mass., USA), polyclonal rabbit anti-phospho-Akt (p-Akt; Cell Signaling Technology Inc.), or polyclonal goat anti- $\alpha$-tubulin (Santa Cruz Biotechnology Inc., Santa Cruz, Calif., USA) overnight at $4^{\circ} \mathrm{C}$. The membranes were then incubated with horseradish peroxidase-conjugated goat anti-rabbit IgG (Cell Signaling Technology Inc.) to detect p-eNOS and p-Akt, and with horseradish peroxidase-conjugated donkey anti-goat IgG (Santa Cruz Biotechnology Inc.) to detect $\alpha$-tubulin, developed with the ECL Plus Detection System (GE Healthcare UK Ltd.), and captured on X-ray film. The signals were scanned and semiquantitated using the Image J program.

\section{Histological Study}

For histological examinations, the kidneys were fixed with $4 \%$ buffered paraformaldehyde for $6 \mathrm{~h}$, embedded in paraffin, and sectioned transversely with a thickness of $4 \mu \mathrm{m}$. By immunostaining for $\alpha$-smooth muscle actin ( $\alpha$-SMA), sections were incubated with monoclonal mouse anti-human $\alpha$-SMA (DAKO, Carpinteria, Calif., USA), according to the manufacturer's protocol [25]. To detect infiltrating macrophages, sections were incubated with monoclonal rat anti-mouse F4/80 (Selotec, Oxford, UK) for $1 \mathrm{~h}$ at room temperature, followed by standard $\mathrm{ABC}$ immunostaining using $\mathrm{ABC}$-alkaline phosphatase kit (Vector, Burlingame, Calif., USA) [24]. A standard point-counting method was used to quantitate the collagen fractional volume in the renal cortical interstitium on Masson's trichrome-stained sections (magnification $\times 400$ ), as previously described [24]. The index of the interstitial collagen fractional volume was defined as the number of trichrome-positive points in every 1,000 points evaluated. The expression of $\alpha$-SMA in the cortical interstitial area was assessed semiquantitatively by immunoreactivity for $\alpha$-SMA, as previously described [25]. The number of infiltrating macrophages was determined by enumerating F4/80-positive cells within the cortical interstitium in 10 randomly selected cortical fields under magnification $(\times 400)$, and the numbers were averaged for each field [24].

\section{Statistical Analysis}

Data are presented as means \pm SEM. Statistical analysis was performed by ANOVA for multiple comparison analysis. Statistical significance was defined as $\mathrm{p}<0.05$. 
Fig. 1. The mRNA expressions of apelin and APJ receptor in the UUO kidneys. Total RNA was extracted from obstructed kidneys at day 7 after UUO and from NOB. mRNA expressions of apelin (a) and APJ receptor (b) were assessed by real-time RTPCR. Data are expressed as means \pm SEM. ${ }^{*} \mathrm{p}<0.05 ;{ }^{* *} \mathrm{p}<$ $0.01 . \mathrm{NS}=$ Not significant.

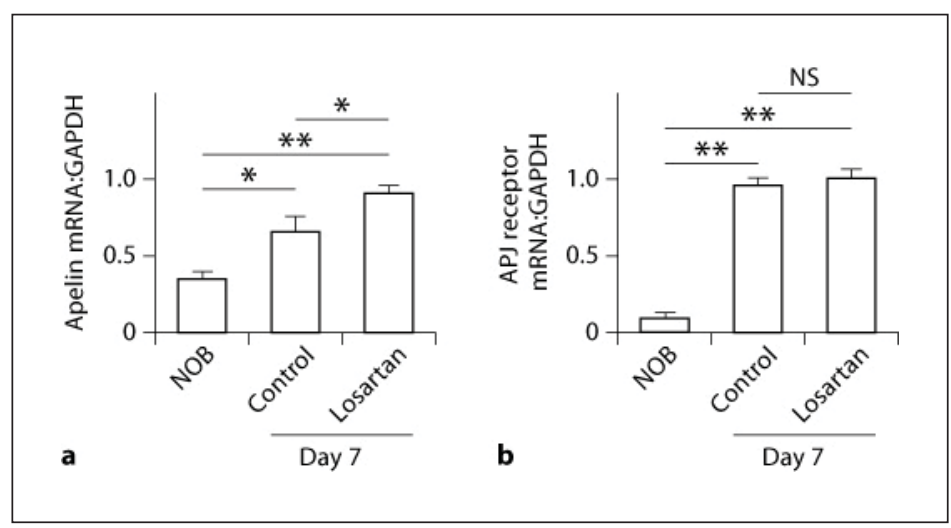

\section{Results}

mRNA Expressions of Apelin and APJ Receptor in the UUO Kidneys

The mRNA expressions of apelin and APJ receptor in the UUO kidneys were both significantly increased at day 7 after UUO compared with those in nonobstructed kidneys $(\mathrm{NOB})$ at day 0 (the ratio to GAPDH mRNA, apelin: $0.37 \pm 0.04$ vs. $0.65 \pm 0.12, \mathrm{p}<0.05$, $\mathrm{n}=6$ in each group; APJ receptor: $0.13 \pm 0.01$ vs. $0.97 \pm 0.03, \mathrm{p}<0.01, \mathrm{n}=6$ ) (fig. $1 \mathrm{a}, \mathrm{b}$ ). Treatment with losartan further significantly enhanced the expression of apelin mRNA compared with that in the control group at day 7 after UUO $(0.90 \pm 0.03, p<0.05$ vs. control group, $n=6$ ) (fig. 1a); however, no significant change was observed in the expression of APJ receptor with losartan treatment $(0.98 \pm 0.03$, not signficant vs. control group, $n=6)($ fig. $1 b)$.

\section{Blood Pressure}

No significant difference was observed in the systolic blood pressure between the groups either at day 0 (before UUO), day 3, or day 7 after UUO (control; losartan; losartan + F13A; losartan + L-NAME groups, respectively; day 0: $108 \pm 4$; $97 \pm 2$; $96 \pm 2$; $98 \pm 4 \mathrm{~mm} \mathrm{Hg}$, not significant among groups; day 3: $97 \pm 3 ; 94 \pm 1 ; 97 \pm 2 ; 95 \pm 1 \mathrm{~mm} \mathrm{Hg}$, not significant among groups; day 7: $102 \pm 3 ; 94 \pm 2 ; 95 \pm 2 ; 93 \pm 6 \mathrm{~mm} \mathrm{Hg}$, not significant among groups; $\mathrm{n}=9$ in the control group, $\mathrm{n}=8$ in the losartan group, $\mathrm{n}=6$ in the losartan + F13A and losar$\tan +\mathrm{L}-\mathrm{NAME}$ groups).

\section{p-eNOS and p-Akt Protein Expressions}

The protein expressions of p-eNOS and p-Akt in the UUO kidneys were assessed by Western blotting and were both increased at day 7 after UUO compared with those in NOB at day 0 (the ratio to $\alpha$-tubulin protein, $\mathrm{p}$-eNOS: $0.20 \pm 0.02$ vs. $0.72 \pm 0.11, \mathrm{p}<0.01, \mathrm{n}=6$ in each group; $\mathrm{p}$-Akt: $0.13 \pm 0.02$ vs. $0.41 \pm 0.03, \mathrm{p}<0.01, \mathrm{n}=6$ in each group). Treatment with losartan further enhanced phosphorylations of both of these proteins (p-eNOS: $2.74 \pm$ $0.18, \mathrm{p}<0.01$ vs. control group, $\mathrm{n}=6$; $\mathrm{p}$-Akt: $0.83 \pm 0.07, \mathrm{p}<0.01$ vs. control group, $\mathrm{n}=6$ ), and cotreatment with F13A, a APJ receptor antagonist, significantly suppressed the effects of losartan on the expressions of both of these proteins (p-eNOS: $0.81 \pm 0.12, p<0.01$ vs. losartan group, $\mathrm{n}=6$; p-Akt: $0.43 \pm 0.03, \mathrm{p}<0.01$ vs. losartan group, $\mathrm{n}=6$ ) (fig. $2 \mathrm{a}-\mathrm{c}$ ).

\section{Interstitial Fibrosis}

Treatment with losartan resulted in a significant decrease in the interstitial collagen index at day 7 after UUO compared with the control group (64 \pm 3 vs. $41 \pm 4 / 1,000$ points, 


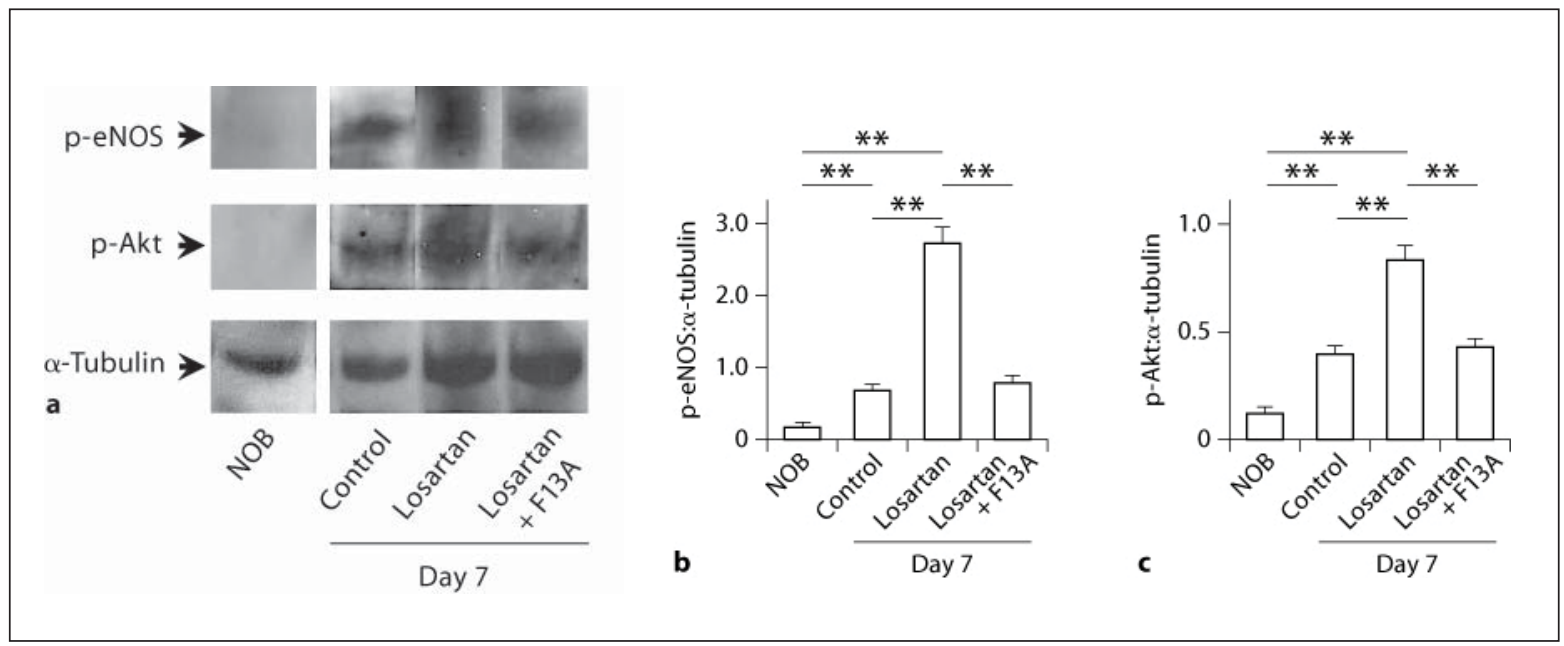

Fig. 2. p-eNOS and p-Akt protein expressions in the UUO kidneys. Whole kidney lysates from obstructed kidneys at day 7 after UUO and from NOB were examined for p-eNOS and p-Akt protein expressions by Western blot analysis (a), and quantitatively analysed as the ratio to $\alpha$-tubulin protein for p-eNOS (b) and p-Akt (c). Data are expressed as means \pm SEM. ${ }^{* *} \mathrm{p}<0.01$.

$\mathrm{p}<0.01, \mathrm{n}=9$ in the control group and $\mathrm{n}=8$ in the losartan group). However, treatment with both losartan + F13A and losartan + L-NAME resulted in significant increases in the interstitial collagen index compared to mice treated with losartan only, and also compared to control mice receiving no treatment $(83 \pm 3$ in the losartan + F13A group, $\mathrm{p}<0.01$ vs. losartan group, $\mathrm{p}<0.05$ vs. control group, $\mathrm{n}=6$; $84 \pm 3$ in the losartan + L-NAME group, $\mathrm{p}<$ 0.01 vs. losartan group, $\mathrm{p}<0.05$ vs. control, $\mathrm{n}=6$ ) (fig. 3a, b).

\section{Myofibroblast Accumulation}

The degree of interstitial myofibroblast accumulation assessed by immunostaining for $\alpha$-SMA was significantly lower in mice treated with losartan compared to that in the control group at day 7 after UUO ( $\alpha$-SMA score; $1.88 \pm 0.09$ vs. $1.32 \pm 0.08, \mathrm{p}<0.01, \mathrm{n}=9$ in the control group and $n=8$ in the losartan group). However, treatment with both losartan + F13A and losartan + L-NAME resulted in significant increases in the interstitial myofibroblast accumulation compared with mice treated with losartan only, and also compared to control mice receiving no treatment $(2.38 \pm 0.14$ in the losartan + F13A group, $\mathrm{p}<0.01$ vs. losartan group, $\mathrm{p}<0.05$ vs. control group, $\mathrm{n}=6 ; 2.43 \pm 0.08$ in the losartan + L-NAME group, $\mathrm{p}<0.01$ vs. losartan group, $\mathrm{p}<0.05$ vs. control group, $\mathrm{n}=6$ ) (fig. $3 \mathrm{a}, \mathrm{c}$ ).

\section{Interstitial Macrophage Infiltration}

The number of F4/80-positive macrophages infiltrating to the interstitium was significantly decreased in mice treated with losartan compared to that in the control group at day 7 after UUO $(24.0 \pm 1.2$ vs. $17.6 \pm 1.5 / \times 400$ field, $\mathrm{p}<0.01, \mathrm{n}=9$ in the control group and $\mathrm{n}=8$ in the losartan group). However, treatment with both losartan + F13A and losartan + L-NAME resulted in significant increases in the number of interstitial macrophages compared with mice treated with losartan only, and also compared to control mice receiving no treatment $(34.4 \pm 1.5$ in the losartan + F13A group, $\mathrm{p}<0.01$ vs. losartan group, $\mathrm{p}<0.01$ vs. control group, $\mathrm{n}=6 ; 36.0 \pm 1.6$ in the losartan $+\mathrm{L}-\mathrm{NAME}$ group, $\mathrm{p}<0.01$ vs. losartan group, $\mathrm{p}<0.01$ vs. control, $\mathrm{n}=6$ ) (fig. 3a, d). 


\begin{tabular}{|c|c|}
\hline Nephron Extra 2012;2:39-47 & \\
\hline $\begin{array}{l}\text { DOI: } 10.1159 / 000337091 \\
\text { Published online: March 7, } 2012\end{array}$ & $\begin{array}{l}\text { (c) } 2012 \text { S. Karger AG, Basel } \\
\text { www.karger.com/nne }\end{array}$ \\
\hline
\end{tabular}
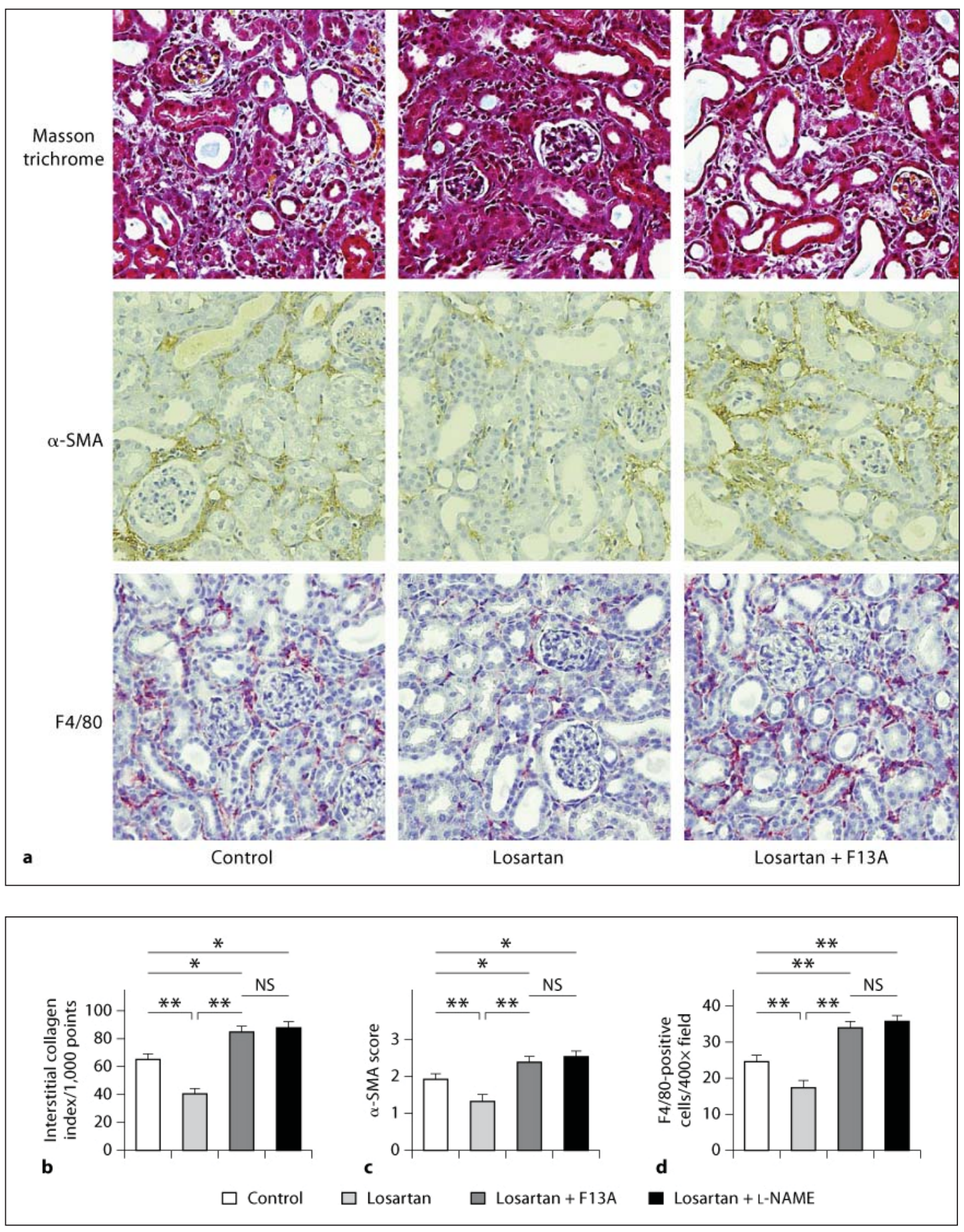

Fig. 3. Histological assessment of interstitial fibrosis, myofibroblast accumulation, and macrophage infiltration in the UUO kidneys. Masson's trichrome staining and immunohistochemical demonstration of myofibroblasts with anti- $\alpha$-SMA antibody, and of macrophages with anti-F4/80 antibody in control mice, in mice treated with losartan, and in mice treated with losartan + F13A at day 7 after UUO (a). Original magnification, $\times 400$. Quantitative analysis of interstitial collagens assessed by the point-counting method on Masson's trichrome-stained sections (b), of interstitial myofibroblast accumulation assessed by $\alpha$-SMA score (c), and of the number of F4/80-positive macrophages infiltrating to the interstitium (d) in control mice, in mice treated with losartan, in mice treated with losartan $+\mathrm{F} 13 \mathrm{~A}$, and in mice treated with losartan + L-NAME at day 7 after UUO. Data are expressed as means \pm SEM. ${ }^{*} \mathrm{p}<0.05$; ${ }^{* *} \mathrm{p}<0.01$. NS $=$ Not significant. 


\section{Discussion}

In this study, we first examined the mRNA expressions of apelin and APJ receptor in the UUO kidneys and found that both apelin and APJ receptor mRNAs were upregulated at day 7 after UUO. Furthermore, treatment with losartan resulted in a significant increase in the apelin mRNA expression, but not in that of the APJ receptor, compared with the control group at day 7 after UUO. These results suggest that the apelin/APJ system is upregulated in the UUO kidney compared with that in the NOB, and that the blockade of the AT-1 receptor results in further upregulation of apelin production in this model. These results are consistent with previous reports showing a counter-regulatory role of the apelin/APJ and Ang II/ AT-1 systems [11-17]. One of the major actions of apelin via the APJ receptor is eNOS production through the Akt/eNOS pathway which causes vasodilation [21] and may exert antifibrotic tissue-protective effects in the UUO kidney $[22,23]$. Thus, we next examined the phosphorylation of the Akt/eNOS pathway in the UUO kidney as well as the pathological changes related to renal interstitial fibrosis.

Our results indicate that phosphorylations of both Akt and eNOS are significantly increased in the UUO kidney compared with NOB at day 7 after UUO, and treatment with losartan results in marked increases in both p-Akt and p-eNOS proteins compared with the control group. F13A is a compound generated by substituting carboxyl-terminal phenylalanine in apelin-13 with alanine residue, and it has been proved to act as a specific antagonist of the APJ receptor [26, 27]. When mice were cotreated with losartan + F13A, the effects of losartan on the activation of the Akt/eNOS pathway were completely abrogated. These results suggest that upregulation of apelin production by the treatment with losartan contributes to the activation of the Akt/eNOS pathway in the UUO kidney. Additionally, although treatment with losartan resulted in alleviated renal interstitial fibrosis, decreased myofibroblast accumulation, and a decreased number of macrophages infiltrating to the interstitium compared with the control group, cotreatment with losartan + F13A completely abrogated all these effects of losartan and even significantly increased the degrees of renal fibrosis, myofibroblast accumulation, and the number of infiltrating macrophages compared with the control group. These results suggest that the alleviative effect of losartan in renal fibrosis in the UUO kidney is, at least in part, due to the action of apelin via the APJ receptor. Furthermore, cotreatment with losartan + L-NAME, a NOS inhibitor, also completely abrogated the effects of losartan and significantly increased the degrees of renal fibrosis, myofibroblast accumulation, and the number of infiltrating macrophages to a level comparable with losar$\tan +$ F13A treatment. These results suggest that increased NO production through the apelin/APJ/Akt/eNOS pathway may, at least in part, contribute to the alleviative effect of losar$\tan$ in UUO-induced renal fibrosis.

The antifibrotic and antiapoptotic effects of NO on the UUO kidney have been reported in several previous studies [22,23]. Dietary arginine supplementation has been shown to improve renal damage including fibrosis and apoptosis in the UUO kidney without affecting renal blood flow and glomerular filtration rate, suggesting increased NO bioavailability [23]. Also in our study, the systolic blood pressures of mice of each experimental group did not significantly differ, and increased NO production may possibly exert antifibrotic tissue-protective effects presumably by improving microcirculation and acting as antiapoptotic in the UUO kidney.

Although the mechanism for the counter-regulatory action of the apelin/APJ system to the renin-angiotensin system is still unclear, growing evidence indicates that apelin mediates opposing actions to Ang II. Apelin attenuates Ang II-induced vasoconstriction through both the NO-dependent and NO-independent pathway $[12,13]$. Besides, in the kidney, apelin has glomerular hemodynamic functions that oppose to Ang II through the release of NO [16]. A 
recent report also showed that apelin inhibits Ang II signaling pathways including extracellular signal-regulated kinase phosphorylation and transcriptional regulation of multiple targets, and that the AT-1 and APJ receptors can physically associate on the cell membrane by forming heterodimers and may influence downstream signaling in a stoichiometric fashion [15]. Furthermore, protective effects of apelin in cardiovascular fibrosis have also been shown more recently in a model of Ang II-induced cardiovascular fibrosis [18] and during the treatment with an angiotensin receptor blocker [17]. However, our study is the first report that shows the role of the apelin/APJ system on the alleviative effect of renal fibrosis during inhibition of the Ang II/AT-1 system.

In summary, we have shown, for the first time, that pharmacological blockade of the Ang II/AT-1 system upregulates the apelin/APJ system, and that the apelin/APJ system contributes to the alleviative effect of renal fibrosis on the UUO kidney via activation of the Akt/ eNOS pathway. Further studies regarding the role of apelin/APJ during the evolution of renal fibrosis and the regulatory mechanism of the apelin/APJ and renin-angiotensin systems are considered necessary. Nevertheless, our study provides insights into the role of Ang II inhibition and, more importantly, raises the possibility of therapeutic options targeting apelin/ APJ as well as the renin-angiotensin system for the treatment of renal fibrosis.

\section{Acknowledgement}

This study was supported in part by a Grant-in-Aid for Scientific Research C from the Japan Society for the Promotion of Science (19591263).

\section{Disclosure Statement}

Losartan used in this experiment was provided by Merck \& Co. Inc.

\section{References}

-1 O’Dowd BF, Heiber M, Chan A, Heng HH, Tsui LC, Kennedy JL, Shi X, Petronis A, George SR, Nguyen T: A human gene that shows identity with the gene encoding the angiotensin receptor is located on chromosome 11. Gene 1993;136:355-360.

-2 Tatemoto K, Hosoya M, Habata Y, Fujii R, Kakegawa T, Zou MX, Kawamata Y, Fukusumi S, Hinuma S, Kitada C, Kurokawa T, Onda H, Fujino M: Isolation and characterization of a novel endogenous peptide ligand for the human APJ receptor. Biochem Biophys Res Commun 1998;251:471-476.

-3 O'Carroll AM, Selby TL, Palkovits M, Lolait SJ: Distribution of mRNA encoding B78/apj, the rat homologue of the human APJ receptor, and its endogenous ligand apelin in brain and peripheral tissues. Biochim Biophys Acta 2000;1492:72-80.

-4 Lee DK, Cheng R, Nguyen T, Fan T, Kariyawasam AP, Liu Y, Osmond DH, George SR, O’Dowd BF: Characterization of apelin, the ligand for the APJ receptor. J Neurochem 2000;74:34-41.

-5 Kawamata Y, Habata Y, Fukusumi S, Hosoya M, Fujii R, Hinuma S, Nishizawa N, Kitada C, Onda H, Nishimura O, Fujino M: Molecular properties of apelin: tissue distribution and receptor binding. Biochim Biophys Acta 2001;1538:162-171.

-6 Medhurst AD, Jennings CA, Robbins MJ, Davis RP, Ellis C, Winborn KY, Lawrie KW, Hervieu G, Riley G, Bolaky JE, Herrity NC, Murdock P, Darker JG: Pharmacological and immunohistochemical characterization of the APJ receptor and its endogenous ligand apelin. J Neurochem 2003;84:11621172 . 
7 Kleinz MJ, Skepper JN, Davenport AP: Immunocytochemical localization of the apelin receptor, APJ, to human cardiomyocytes, vascular smooth muscle and endothelial cells. Regul Pept 2005;126: 233-240.

8 Falcao-Pires I, Leite-Moreira AF: Apelin: a novel neurohumoral modulator of the cardiovascular system: pathophysiologic importance and potential use as a therapeutic target. Rev Port Cardiol 2005;24:1263-1276.

-9 Kleinz MJ, Davenport AP: Emerging roles of apelin in biology and medicine. Pharmacol Ther 2005; 107:198-211.

-10 Masri B, Knibiehler B, Audigier Y: Apelin signalling: a promising pathway from cloning to pharmacology. Cell Signal 2005;17:415-426.

-11 Ashley E, Chun HJ, Quertermous T: Opposing cardiovascular roles for the angiotensin and apelin signaling pathways. J Mol Cell Cardiol 2006;41:778-781.

-12 Gurzu B, Petrescu BC, Costuleanu M, Petrescu G: Interactions between apelin and angiotensin II on rat portal vein. J Renin Angiotensin Aldosterone Syst 2006;7:212-216.

-13 Zhong JC, Huang Y, Yung LM, Lau CW, Leung FP, Wong WT, Lin SG, Yu XY: The novel peptide apelin regulates intrarenal artery tone in diabetic mice. Regul Pept 2007;144:109-114.

- 14 Ishida J, Hashimoto T, Hashimoto Y, Nishiwaki S, Iguchi T, Harada S, Sugaya T, Matsuzaki H, Yamamoto R, Shiota N, Okunishi H, Kihara M, Umemura S, Sugiyama F, Yagami K, Kasuya Y, Mochizuki N, Fukamizu A: Regulatory roles for APJ, a seventransmembrane receptor related to AT1, in blood pressure in vivo. J Biol Chem 2004;279:26274-26279.

-15 Chun HJ, Ali ZA, Kojima Y, Kundu RK, Sheikh AY, Agrawal R, Zheng L, Leeper NJ, Pearl NE, Patterson AJ, Anderson JP, Tsao PS, Lenardo MJ, Ashley EA, Quertermous T: Apelin signaling antagonizes Ang II effects in mouse models of atherosclerosis. J Clin Invest 2008;118:3343-3354.

-16 Hus-Citharel A, Bouby N, Frugière A, Bodineau L, Gasc JM, Llorens-Cortes C: Effect of apelin on glomerular hemodynamic function in the rat kidney. Kidney Int 2008;74:486-494.

- 17 Fukushima H, Kobayashi N, Takeshima H, Koguchi W, Ishimitsu T: Effects of olmesartan on apelin/ APJ and Akt/eNOS pathway in Dahl rats with end-stage heart failure. J Cardiovasc Pharmacol 2010; 55:83-88.

$\checkmark 18$ Siddiquee K, Hampton J, Khan S, Zadory D, Gleaves L, Vaughan DE, Smith LH: Apelin protects against angiotensin II-induced cardiovascular fibrosis and decreases plasminogen activator inhibitor type-1 production. J Hypertens 2011;29:724-731.

19 Brenner BM: Remission of renal disease: recounting the challenge, acquiring the goal. J Clin Invest 2002;110:1753-1758.

-20 Bascands JL, Schanstra JP: Obstructive nephropathy: insights from genetically engineered animals. Kidney Int 2005;68:925-937.

-21 Tatemoto K, Takayama K, Zou MX, Kumaki I, Zhang W, Kumano K, Fujimiya M: The novel peptide apelin lowers blood pressure via a nitric oxide-dependent mechanism. Regul Pept 2001;99:87-92.

-22 Miyajima A, Chen J, Poppas DP, Vaughan ED Jr, Felsen D: Role of nitric oxide in renal tubular apoptosis of unilateral ureteral obstruction. Kidney Int 2001;59:1290-1303.

-23 Ito K, Chen J, Seshan SV, Khodadadian JJ, Gallagher R, El Chaar M, Vaughan ED Jr, Poppas DP, Felsen D: Dietary arginine supplementation attenuates renal damage after relief of unilateral ureteral obstruction in rats. Kidney Int 2005;68:515-528.

-24 Nishida M, Fujinaka H, Matsusaka T, Price J, Kon V, Fogo AB, Davidson JM, Linton MF, Fazio S, Homma T, Yoshida H, Ichikawa I: Absence of angiotensin II type 1 receptor in bone marrow-derived cells is detrimental in the evolution of renal fibrosis. J Clin Invest 2002;110:1859-1868.

-25 Fujinaka H, Miyazaki Y, Matsusaka T, Yoshida H, Fogo AB, Inagami T, Ichikawa I: Salutary role for angiotensin in partial urinary tract obstruction. Kidney Int 2000;58:2018-2027.

-26 Lee DK, Saldivia VR, Nguyen T, Cheng R, George SR, O’Dowd BF: Modification of the terminal residue of apelin-13 antagonizes its hypotensive action. Endocrinology 2005;146:231-236.

-27 Tiani C, Garcia-Pras E, Mejias M, de Gottardi A, Berzigotti A, Bosch J, Fernandez M: Apelin signaling modulates splanchnic angiogenesis and portosystemic collateral vessel formation in rats with portal hypertension. J Hepatol 2009;50:296-305. 